\title{
Recommendations of the Polish Medical Society of Radiology and the Polish Society of Neurology for a protocol concerning routinely used magnetic resonance imaging in patients with multiple sclerosis
}

\author{
Marek Sąsiadek1, Marcin Hartel ${ }^{2}$, Małgorzata Siger ${ }^{3}$, Katarzyna Katulska ${ }^{4}$, Agata Majos ${ }^{5}$, Ewa Kluczewska ${ }^{6}$, \\ Halina Bartosik-Psujek ${ }^{7}$, Alina Kułakowska ${ }^{8}$, Agnieszka Słowik ${ }^{9}$, Barbara Steinborn ${ }^{10}$, Monika Adamczyk-Sowa ${ }^{11}$, \\ Alicja Kalinowska ${ }^{12}$, Ewa Krzystanek ${ }^{13}$, Robert Bonek ${ }^{14}$, Zbigniew Serafin ${ }^{15}$, Jarosław Sławek ${ }^{16}$, \\ Przemysław Nowacki ${ }^{17}$, Adam Stępieńn ${ }^{18}$, Sergiusz Jóżwiak ${ }^{19}$, Konrad Rejdak ${ }^{20}$, Krzysztof Selmaj ${ }^{21}$, Jerzy Walecki22
}

'Department of General and Interventional Radiology and Neuroradiology, Wroclaw Medical University, Wroclaw, Poland

${ }^{2}$ Medical Diagnostic Centre Voxel, Katowice, Poland

${ }^{3}$ Department of Neurology, Medical University of Lodz, Lodz, Poland

${ }^{4}$ Department of Neuroradiology, Poznan University of Medical Sciences, Poznan, Poland

${ }^{5}$ Department of Radiological and Isotopic Diagnosis and Therapy, Medical University of Lodz, Lodz, Poland

${ }^{6}$ Department and Institute of Medical Radiology and Radiodiagnostics in Zabrze, Medical University of Silesia in Katowice, Poland

${ }^{7}$ Faculty of Medicine, University of Rzeszow, Rzeszow, Poland

${ }^{8}$ Department of Neurology, Medical University of Bialystok, Bialystok, Poland

${ }^{9}$ Department of Neurology, Jagiellonian University Medical College, University Hospital in Krakow, Krakow, Poland

${ }^{10}$ Department of Developmental Neurology, Poznan University of Medical Sciences, Poznan, Poland

${ }^{11}$ Department of Neurology in Zabrze, Medical University of Silesia, Zabrze, Poland

${ }^{12}$ Department of Neurology, Division of Neurochemistry and Neuropathology, Poznan University of Medical Sciences, Poznan, Poland

${ }^{13}$ Department of Neurology, School of Medicine in Katowice, Medical University of Silesia, Katowice, Poland

${ }^{14}$ Department of Neurology and Clinical Neuroimmunology, Regional Specialist Hospital, Grudziądz, Poland

${ }^{15}$ Department of Radiology and Diagnostic Imaging, Nicolaus Copernicus University, Collegium Medicum, Bydgoszcz, Poland

${ }^{16}$ Department of Neurology, St. Adalbert Hospital, “Copernicus" Ltd., Gdańsk, Poland

${ }^{17}$ Department of Neurology, Pomeranian Medical University, Szczecin, Poland

${ }^{18}$ Department of Neurology, Military Institute of Medicine, Warsaw, Poland

${ }^{19}$ Department of Paediatric Neurology, Warsaw Medical University, Warsaw, Poland

${ }^{20}$ Department of Neurology, Medical University of Lublin, Lublin, Poland

${ }^{21}$ Department of Neurology, Laboratory of Neuroimmunology, Faculty of Medicine, University of Warmia and Mazury, Olsztyn, Poland

${ }^{22}$ Department of Radiology, Medical Centre for Postgraduate Education, Warsaw, Poland 


\section{Abstract}

Magnetic resonance imaging (MRI) is a widely used method for the diagnosis of multiple sclerosis (MS) that is essential for the detection and follow-up of the disease.

The Polish Medical Society of Radiology (PLTR) and the Polish Society of Neurology (PTN) present the second version of their recommendations for investigations routinely conducted in MRI departments in patients with multiple sclerosis. This version includes new data and practical comments for electroradiology technologists and radiologists. The recommended protocol aims to improve the MRI procedure and, most importantly, to standardise the method of conducting scans in all MRI departments. This is crucial for the initial diagnostics necessary for establishing a diagnosis, as well as for MS patient monitoring, which directly translates into significant clinical decisions.

MS is a chronic immune mediated inflammatory demyelinating disease of the central nervous system (CNS), the aetiology of which is still unknown. The nature of the disease lies in a CNS destruction process disseminated in time and space. MRI detects focal lesions in the white and grey matter with high sensitivity (although with significantly lower specificity in the latter). It is also the best tool to assess brain atrophy in patients with MS in terms of grey matter volume and white matter volume as well as local atrophy (by measuring the volume of thalamus, corpus callosum, subcortical nuclei, and hippocampus) as parameters that correlate with disability progression and cognitive dysfunctions. Progress in MR techniques, as well as advances in postprocessing the obtained data, has driven the dynamic development of computer programs that allow for a more repeatable assessment of brain atrophy in both cross-sectional and longitudinal studies. MRI is unquestionably the best diagnostic tool available to follow up the course of the disease and support clinicians in choosing the most appropriate treatment strategy for their MS patient. However, to diagnose and follow up MS patients on the basis of MRI in accordance with the latest standards, the MRI study must adhere to certain quality criteria. Such criteria are the subject of this paper.

Key words: multiple sclerosis, magnetic resonance imaging, imaging protocol, guidelines.

\section{Recommendations for MRI in MS patients on MRI scanners with field strength at least $1.5 \mathrm{~T}$}

\section{General comments}

Patients with multiple sclerosis (MS) are recommended to undergo magnetic resonance imaging (MRI) with an intravenous administration of a paramagnetic contrast medium (gadolinium) solely as a part of initial diagnostics.

A follow-up gadolinium-enhanced MRI with the use of macrocyclic contrast agents is recommended only in cases of a clinical progression of the disease, or if the need arises for another differential diagnosis of MS, or in another clinically justified situation. The retention characteristics of each gadolinium-based contrast agent (GBCA) should be considered in all patients; linear GBCAs have been shown to result in greater retention, and retention for longer, than macrocyclic GBCAs. This is why the use of linear contrast agents based on gadolinium (GBCA) is not recommended in following up the treatment of MS in clinically and radiologically stable patients, due to the potential occurrence of long-term side effects associated with the accumulation of contrast agent within the central nervous system (CNS).

Patients with clinically isolated syndrome (CIS) or suspected MS should undergo:

- Head MRI before and after an intravenous administration of a contrast agent (Table 1).

TABLE 1. Head magnetic resonance imaging protocol

\begin{tabular}{|c|c|}
\hline Parameters & Description \\
\hline Electromagnetic field & Images should be of good quality with an appropriate SNR value and resolution $(\leq 1 \times 1 \mathrm{~mm})$ \\
\hline Reference setting & $\begin{array}{l}\text { When setting the scanning plane, use a line parallel to the lower edges of the rostrum and splenium of the corpus callosum; also to have } \\
\text { an identical angulation of the planned slices to that of the slices in the previous study (Figure 1) }\end{array}$ \\
\hline Scanning range & Whole brain scanned \\
\hline Slice thickness and gaps & $\leq 3 \mathrm{~mm}$, with no gaps (for 2D and 3D acquisitions) \\
\hline Basic sequences & $\begin{array}{l}\text { 1. 3DT1 axial isotropically } \\
\text { 2. T2 axial } \\
\text { 3. DWI axial (with ADC map) } \\
\text { Administration of a contrast medium }{ }^{1} \text { ( } T 1 \text { sequence } 5-10 \text { minutes after administration) } \\
\text { 4. FLAIR + C sagittal } \\
\text { 5. FLAIR + C axial } \\
\text { 6. } 3 D T 1+C \text { axial isotropically }{ }^{2} \\
\text { 'The recommended dose of a contrast medium is } 0.1 \mathrm{mmol} / \mathrm{kg} \text { body mass; see General comments. } \\
\text { 2It is recommended to perform and record sagittal reconstructions and archive them on a CD (and in the PACS system if available), as an integral part of the examination. }\end{array}$ \\
\hline Optional sequences & $\begin{array}{l}\text { 1. PD } \\
\text { 2. SWI - to identify central veins in lesions and microbleedings } \\
\text { 3. DIR - to evaluate cortical and subcortical foci }\end{array}$ \\
\hline
\end{tabular}

SNR - signal-to-noise ratio, 2D - two-dimensional, 3D - three-dimensional, DWI - diffusion-weighted imaging, ADC - apparent diffusion coefficient, FLAIR - fluid-attenuated inversion recovery sequence, $+C$ - postcontrast, PACS - picture archiving and communication system, PD - proton density, SWI - susceptibility weighted imaging, DIR - double inversion recovery 


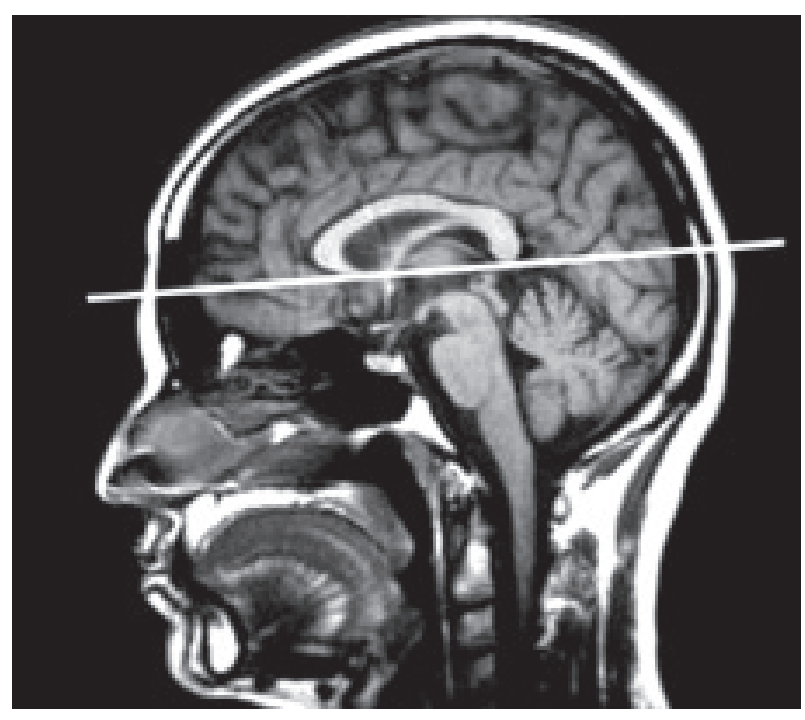

Figure 1. A pre-specified reference line parallel to the corpus callosum

- It is recommended that an additional scan of the cervical and thoracic sections of the spinal cord (in accordance with the referral/indication of the neurologist) is performed after an intravenous administration of a contrast agent, especially when head MRI does not meet the diagnostic criteria or when clinical symptoms suggest the lesions are located in the spinal cord (Table 2). Ideally, MRI studies of the head and the cervical/thoracic spine should be performed within a single MRI imaging session. MRI of the spinal cord is particularly important in the diagnosis of primary progressive MS according to the current disease diagnosis criteria (Table 3).

In patients with multifocal damage to the nervous system involving symptomatology associated with both brain and spinal cord impairment, in order to shorten the diagnostic time, (and according to the referring neurologist's recommendations) it is possible to perform simultaneously an MRI of the head and of a selected section of the spinal cord using a combined protocol (Table 4).

Recommendations for disease progression follow-up based on MRI:

- Head MRI to show new/enlarging demyelinating lesions (Table 1) at least every 12 months during the initial years of treatment, but possibly less frequently later in patients with complete clinical stability.

TABLE 2. Spinal cord section magnetic resonance imaging protocol

\begin{tabular}{|c|c|}
\hline Parameters & Description \\
\hline Electromagnetic field & Images should be of good quality with an appropriate SNR value and resolution $(\leq 1 \times 1 \mathrm{~mm})$ \\
\hline Scanning range & Spinal cord section scanned \\
\hline Slice thickness and gaps & $\begin{array}{l}\text { Sagittal: } \leq 3 \mathrm{~mm} \text {, no gaps (for } 2 \mathrm{D} \text { and 3D) } \\
\text { Axial: } 3 \mathrm{~mm} \text {, no gaps }\end{array}$ \\
\hline Basic sequences & $\begin{array}{l}\text { 1. T2 sagittal } \\
\text { 2. T1 sagittal } \\
\text { Administration of a contrast medium } 11 \text { (T1 sequence } 5-10 \text { minutes after administration) } \\
\text { 3. STIR/T2 FAT-SAT, PD or PST1-IR + C sagittal } \\
\text { 4. T2+C axial at the level of the lesions visible in sagittal sequences } \\
\text { 5. } T 1+C \text { sagittal } \\
\text { 6. T1+C axial } \\
\text { 'The recommended dose of a contrast medium is } 0.1 \mathrm{mmol} / \mathrm{kg} \text { body mass; see General Comments }\end{array}$ \\
\hline Optional sequences & $\begin{array}{l}\text { 1. T2 coronal at the level of the lesions visible in the other sequences } \\
\text { 2.3DT1 sagittal (to assess spinal cord atrophy) }\end{array}$ \\
\hline
\end{tabular}

SNR - signal-to-noise ratio, 2D - two-dimensional, 3D - three-dimensional, STIR - short tau inversion recovery, FAT-SAT - fat saturation, PD - proton density, PST1-IR - phase-sensitive T1-weighted inversion-recovery, $+\mathrm{C}-$ postcontrast

TABLE 3. 2017 McDonald criteria for multiple sclerosis diagnosis

\begin{tabular}{|l|l|}
\hline Clinical presentation & Additional criteria required for diagnosis \\
\hline Minimum two relapses, clinical signs from two foci & Not required* \\
\hline Minimum two relapses, clinical signs from one focus & DIS damage to the nervous system on MRI or another relapse of different clinical location \\
\hline One relapse, clinical signs from two or more foci & $\begin{array}{l}\text { DIT damage to the nervous system on MRI, or presence of specific oligoclonal bands } \\
\text { in cerebrospinal fluid (absent in serum) or subsequent relapse }\end{array}$ \\
\hline $\begin{array}{l}\text { One relapse, clinical signs from one focus } \\
\text { (isolated CNS damage) }\end{array}$ & $\begin{array}{l}\text { DIS and DIT damage to the nervous system on MRI, or presence of specific oligoclonal bands } \\
\text { (absent in serum) in cerebrospinal fluid }\end{array}$ \\
\hline Primary progressive MS & $\begin{array}{l}\text { One year of neurological disability progression diagnosed prospectively or retrospectively, } \\
\text { and two of the following conditions fulfilled: } \\
\text { 1. MRI dissemination in space (but one and not two typical locations required) } \\
\text { 2. MRI dissemination in space in the spinal cord (minimum of two lesions) } \\
\text { 3. Positive CSF test (presence of oligoclonal bands, absent in serum, and/or elevated immunoglobulin index) }\end{array}$ \\
\hline
\end{tabular}

*Once other possible causes of symptoms have been excluded; in practice, every patient with suspected MS should undergo as a minimum an MRI of the head and spinal cord as well as a lumbar puncture 
TABLE 4. Head and spinal cord section magnetic resonance imaging protocol (combined)

\begin{tabular}{|c|c|}
\hline Parameters & Description \\
\hline Electromagnetic field & Images should be of good quality with an appropriate SNR value and resolution $(\leq 1 \times 1 \mathrm{~mm})$ \\
\hline Scanning range & Brain and cervical spinal cord scanned \\
\hline Slice thickness and gaps & Head and spinal cord (sagittal) $\leq 3 \mathrm{~mm}$, no gaps (for 2D and 3D). Spinal cord (axial): $3 \mathrm{~mm}$, no gaps \\
\hline Basic sequences & $\begin{array}{l}\text { 1. Use protocol prior to the administration of a contrast medium for the head. } \\
\text { Administration of a contrast medium' (T1 sequence } 5-10 \text { minutes after administration) } \\
\text { 2. FLAIR+C sagittal (head) } \\
\text { 3. FLAIR+C axial (head) } \\
\text { 4. 3DT1+C axial isotropically (head) } \\
\text { 5. T2+C sagittal (spinal cord section) } \\
\text { 6. STIR/T2 FAT-SAT, PD or PST1-IR + ( sagittal (spinal cord section) } \\
\text { 7. T2+C axial at the level of the lesions visible in sagittal sequences (spinal cord section) } \\
\text { 8. T1+C sagittal (spinal cord section) } \\
\text { 9. T1+C axial (spinal cord section) } \\
\text { 'The recommended dose of a contrast medium is } 0.1 \mathrm{mmol} / \mathrm{kg} \text { body mass; see General Comments. } \\
\text { 2It is recommended to perform and record sagittal reconstructions and archive on a CD (and in the PACS system if available), as an integral part of the examination. }\end{array}$ \\
\hline Optional sequences & As in the specified protocols of the head and spinal cord. \\
\hline
\end{tabular}

SNR - signal-to-noise ratio, 2D - two-dimensional, 3D - three-dimensional, FLAIR - fluid-attenuated inversion recovery sequence, STIR - short tau inversion recovery, FAT-SAT - fat saturation, PD - proton density, PST1-IR - phase-sensitive T1-weighted inversion-recovery, $+C$ - postcontrast, PACS - picture archiving and communication system

- Cervical and/or thoracic spinal cord scan is recommended, according to the neurologist's referral/indication.

For patients under the age of 18 , the MRI protocol for brain and spinal cord examination remains unchanged; examination is the same as in the adult population.

\section{Brain MRI protocol for MS patients}

In order to use the same scanning planes during the follow-up examinations, it is recommended to achieve slices in the true midline plane.

For this purpose, once three localisation slices have been performed, five slices with a thickness of $3 \mathrm{~mm}$ should be planned as accurately as possible in the sagittal plane on T2-weighted images.

The planned slices should be set parallel to the longitudinal cerebral fissure using the localisation slices, in the transverse and frontal planes. The third of these five slices should pass through the median fissure as accurately as possible.

Cross-sectional slices should be set on the thereby obtained midline slice in parallel to the lower limits of the rostrum (anterior commissure - AC) and splenium of the corpus callosum (posterior commissure - PC), according to the AC-PC reference line (Figure 1).

Note: During the follow-up examinations, when the scanning plan is set in the reference to the corpus callosum, it is vital to compare the angulation of the planned slices with the angulation of the slices in the previous study.

Some scanners do not have the possibility of remembering a single slice; in such case the slice should be carefully defined as shown in Figure 1.

Note:

1. The direction of scanning in axial scans must be upwards, whereas in sagittal scans it must be from right to left (also when scanning spinal cord).
2. Gaps between slices should be as small as possible (we propose $0.3 \mathrm{~mm}$, i.e. $10 \%$ slice thickness or no gap).

3. 3DT1 - we recommend performing this sequence first, to avoid motion artifacts in the course of the examination. This sequence allows for precise volumetric evaluation of the brain.

4. Both FLAIR sequences should be performed after the administration of a contrast medium, to delay the onset of $\mathrm{T} 1+\mathrm{C}$ acquisition (within the range of 5-10 minutes) in order to achieve better contrast enhancement. A contrast medium does not lessen the quality of FLAIR images. At the same time, the patient's time spent in the scanner is used optimally. The possible enhancement of cortical demyelinating lesions or leptomeninges does not affect study interpretation, because enhancement is in any case assessed on the basis of T1-weighted sequences.

5. If scanning with 3DT2 and 3DFLAIR sequences, if possible they should be done in the sagittal plane and should be used with subsequent cross-reconstruction with $3 \mathrm{~mm}$ slices in the plane set up to the lower edge of the corpus callosum. When scanning in this way, clinicians should consider conducting the 3DT2 sequence scan after a contrast medium injection, so as to ensure an appropriate delay in $\mathrm{T} 1$ acquisition.

6. If software which automatically determines the angulation/range of the layers can be used, as in the previous examination, such a function is recommended.

A radiological report should include standard terminology used in brain assessment.

Description of focal lesions:

1. Location (supratentorial region: cortical, juxtacortical, central white matter, periventricular, infratentorial, corpus callosum, brainstem, spinal cord).

2. Size - the range of the longest dimension provided i.e. from-to. In the case of multiple lesions, report the size of 
TABLE 5. Disseminated in space (DIS) and disseminated in time (DIT) damage to the nervous system according to the 2017 McDonald criteria

\section{Damage to the central nervous system DIS Damage to the central nervous system DIT}

Minimum one T2 lesion present in at least two 0 ccurrence of new lesions on T2-weighted images and/or contrast-enhanced lesions on a subsequent

typical locations:

1. Juxtacortical/cortical

2. Periventricular

3. Infratentorial

4. Spinal cord MRI scan compared to a reference examination, regardless of the time since the baseline examination, or

concomitant occurrence of contrast-enhancing and non-enhancing lesions, regardless of the time of this examination in relation to the time of the onset of neurological signs and symptoms (may also be a basic examination),

or

immunological equivalent of radiological dissemination over time: confirmation of the presence of specific oligoclonal bands (absent in serum) in the cerebrospinal fluid.

the largest lesion only (in mm). According to the current definition, demyelinating lesions are defined as lesions of $\geq 3 \mathrm{~mm}$ in diameter.

3. The number of demyelinating lesions - specify according to the following scheme: $1,2,3-8, \geq 9$.

4. The nature of the lesion, i.e. specify whether the appearance is typical for MS demyelination, or whether differential diagnosis is required, e.g. ischaemic lesion.

5. Whether the lesions are disseminated in space (DIS) and meet the 2017 McDonald criteria (Table 3 and Table 5).

6. Comparison with the previous head and spinal cord MRI (if available). In patients with suspected MS, comparison with the previous MRI to assess disease activity and eligibility for treatment. In on-treatment patients, comparison with the previous examination and baseline examination performed prior to treatment initiation.

7. Activity assessment, i.e. the number of contrast-enhancing foci in the current examination and the number of new/ enlarging lesions compared to the baseline and the previous examination. Please specify according to the scheme: $1,2,3-8, \geq 9$.
8. Assessment of brain atrophy. We recommend avoiding expressions such as "brain atrophy" or "cerebral atrophy" in the report. If possible, provide current whole brain volume, grey matter volume, white matter volume, corpus callosum volume, and the volume of the right and left thalami.

There is a growing need for volumetry assessment in MS patient imaging, because it potentially can change treatment decisions. Many important factors influence volumetric calculations, including acquisition parameters, intrascanner variability, pseudoatrophy, and patient hydration or movement. Having appropriate 3DT1 sequences in the protocol makes it possible to use software available on the market that can measure patient atrophy over time. Volumetric applications differ in the range of output results, and cost is another very important factor. This is why volumetric analysis of the brain should be performed using certified software, and preferably by a central institution in order to standardise results.

\section{Conflict of interest}

The authors report no conflict of interest.

\section{References}

1. Rovira A, Wattjes MP, Tintore M, et al. MAGNIMS consensus guideliness on the use of MRI in multiple sclerosis - clinical implementation in the diagnostic process. Nat Rev Neurol 2015; 11: 471-482.

2. Miller DH, Filippi M, Fazekas F, et al. Role of magnetic resonance imaging within diagnostic criteria for multiple sclerosis. Ann Neurol 2004; 56: 273-278. doi: 10.1002/ana.20156.

3. Simon JH, Traboulsee A, Coyle PK, et al. Standardized MR imaging protocol for multiple sclerosis: consortium of MS centers consensus guidelines. AJNR Am J Neuroradiol 2006; 27: 455-461.

4. Polaman CH, Reingold SC, Banwell B, et al. Diagnostic criteria for multiple sclerosis: 2010 revisions to McDonald criteria. Ann Neurol 2011; 69: 2292-2302.

5. Alroughani R, Al Hashel J, Lamdhade S, et al. Predictors of conversion to multiple sclerosis in patients with clinical isolated syndrome using the 2010 revised McDonald criteria. ISRN Neurol 2012; 2012: 792192.

6. Kelly S, Kinsella K, Duggan M, et al. A proposed modification to the McDonald 2010 criteria for the diagnosis of primary progressive multiple sclerosis. Mult Scler 2013; 19: 1095-1100.

7. Traboulsee A, Simon JH, L Stone, et al. Revised recommendations of the consortium of MS centers task force for a standardized MRI protocol and clinical guidelines for the diagnosis and follow-up of multiple sclerosis. AJNR Am J Neuroradiol 2016; 37: 394-401.

8. Thompson A, Bandwel B, Barkhof F, et al. Diagnosis of multiple sclerosis: 2017 revisions of the McDonald criteria. Lancet Neurol 2018; 17: 162-173.

9. De Stefano N, Airas L, Grigoriadis N, et al. Clinical relevance of brain volume measures in multiple sclerosis. CNS Drugs 2014; 28: 147-156.

10. Zivadinov R, Jakimovski D, Gandhi S, et al. Clinical relevance of brain atrophy assessment in multiple sclerosis. Implications for its use in a clinical routine. Expert Rev Neurother 2016; 16: 777-793.

11. Zivadinov R, Dwyer MG, Bergsland N. Brain atrophy measurements should be used to guide therapy monitoring in MS - YES. Mult Scler 2016; 22: 1522-1524.

12. Sąsiadek M, Katulska K, Majos A, et al. Guidelines of the Polish Medical Society of Radiology for the routinely used MRI protocol in patients with multiple sclerosis. Neurol Neurochir Pol 2018; 52: 638-642.

13. Sastre-Garriga J, Pareto D, Battaglini M, et al. MAGNIMS consensus recommendations on the use of brain and spinal cord atrophy measures in clinical practice. Nat Rev Neurol 2020; 16: 171-182. 